\title{
Hydration Layer-mediated Pairwise Interaction of Nanoparticles resolved by in situ TEM
}

Utkarsh Anand ${ }^{1,2,3,4}$, Utkur Mirsaidov ${ }^{1,2,3,4}$

1. Department of Physics, National University of Singapore, 2 Science Drive 3, Singapore, 117551.

2. Centre for BioImaging Sciences, Department of Biological Sciences, National University of Singapore, Science Drive 4, Singapore, 117543.

3. Centre for Advanced 2D Materials and Graphene Research Centre, National University of Singapore, 6 Science Drive 2, Singapore 117546.

4. $\quad$ NanoCore, 4 Engineering Drive 3, National University of Singapore, Singapore 117576.

Hydration forces play an important role in various physical, chemical and biological processes. They are responsible for colloidal stability, lubrication, crystallization, and interactions between biological molecules, etc [1]. From earlier studies conducted at a macro and micro scale, it is known that these shortrange repulsive forces may stall the approach of interacting surfaces [2]. However, the role of repulsive hydration forces on the interaction dynamics between nanoparticles remains unresolved. Here, we use in situ TEM imaging [3-5] and new image processing algorithms we visualize and quantify the pairwise interactions between gold nanoparticles in water and show that: 1) hydration monolayer around each gold nanoparticle is largely responsible for exponentially increasing hydration repulsion, and 2) the balance between van der Waals (vdW) force and hydration force gives rise to a transient nanoparticle pair [6].

We track the separation between multiple interacting gold nanoparticle pairs in water and find that when the separation between two interacting gold nanoparticle surfaces is two water molecules thick, they form a transient stable pair. When thermal fluctuations drain the water between nanoparticle surfaces, hydration forces vanish and as a result of attractive vdW these nanoparticles jump to contact. The implications of our results for nanoparticle self-assembly will also be discussed [7].

\section{References:}

[1] Parsegian, V. A.; Zemb, T. Curr. Opin. Colloid Interface Sci. 16 (2011) p. 618.

[2] Israelachvili, J. et al. Nature, 379 (1996) p. 219.

[3] Williamson, M. J. et al. Nat Mater, 2 (2003) p. 532.

[4] Zheng, H. et al. Science, 324 (2009) p. 1309.

[5] Ross, F. M. Science 2015, 350 (2015) p. 1490.

[6] Anand, U. et al. Nano Letters, 16 (2016) p. 786.

[7] This work was supported by a Young Investigator Award (NUSYIA-FY14-P17) from the National University of Singapore and the Singapore National Research Foundation's Competitive Research Program funding (NRF-CRP9-2011-04). 

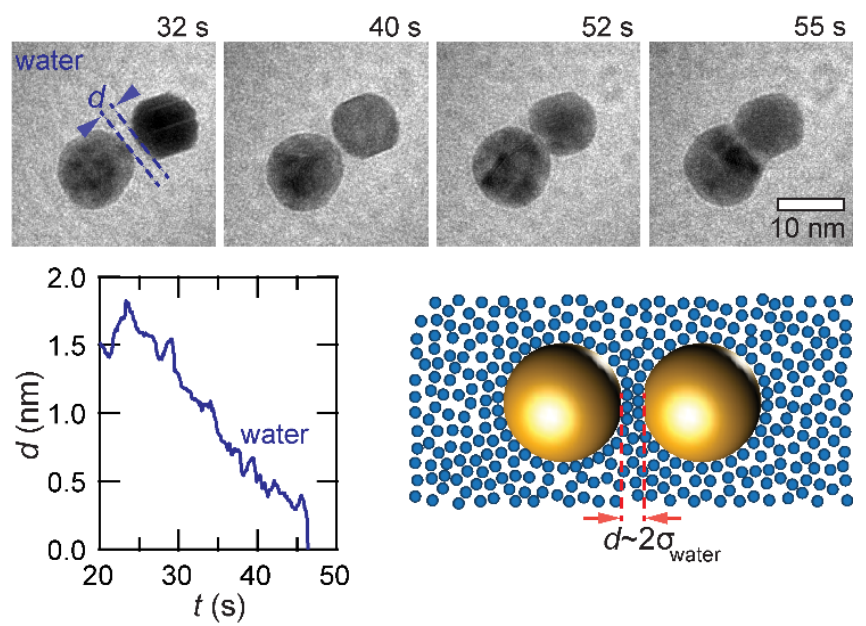

Figure 1. Pairwise interaction of gold nanoparticles. Top: Time-series TEM images of two gold nanoparticles undergoing coalescence in water. Bottom-left: Separation $d$ between the two interacting gold nanoparticles; jump-to-contact occurs at a separation of $5 \AA$. Bottom-right: The corresponding schematic of these nanoparticle interactions in water (blue spheres-water molecules, orange sphere-gold nanoparticle) that arise when gold nanoparticles come into contact with each other with their one-watermolecule-thick hydration layer.

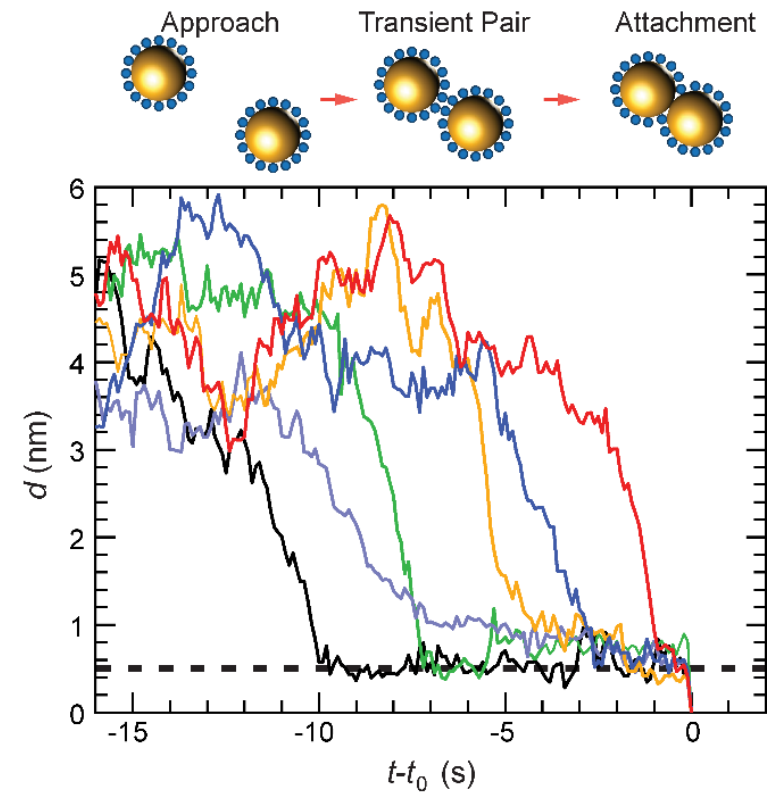

Figure 2. Pairwise separation between interacting gold nanoparticles in water. The plots of the pairwise separations between nanoparticles show the approach down to a pairwise separation of $\sim 0.5 \mathrm{~nm}$ separation set by the combined thickness of the hydration layer of each nanoparticle, at which point the these nanoparticles form a sterically stabilized transient pair. Once the surface-bound water molecules between nanoparticles are drained, the nanoparticle surfaces come into contact at $t-t_{0}=0 \mathrm{~s}$, and coalesce, as schematically illustrated in the top panel. The dashed line shows the spacing which corresponds to two diameters of water molecules. 